\title{
Exploring Optimal Architecture of Multi-layered Feed- forward (MLFNN) as Bidirectional Associative Memory (BAM) for Function Approximation
}

\author{
Manisha Singh \\ M.Tech. (CSE) student \\ Noida Institute of Engineering \& Technology \\ Greater Noida, UP, India
}

\author{
Somesh Kumar \\ Professor \\ Noida Institute of Engineering \& Technology \\ Greater Noida, UP, India
}

\begin{abstract}
Function approximation is an instance of supervised learning which is one of the most studied topics in machine learning, artificial neural networks, pattern recognition, and statistical curve fitting. In principle, any of the methods studied in these fields can be used in reinforcement learning. Multi-layered feed-forward neural networks (MLFNN) have been extensively used for the purpose of function approximation. Another class of neural networks, BAM, has also been studied and experimented for pattern mapping problems and many variations have been reported in literature. In the present study the application of back propagation algorithm to MLFNN has been proposed in such a way that feed-forward architecture behaves like BAM. Various architectures consisting of fourlayers have been explored in quest of finding the optimal architecture for the example function.
\end{abstract}

\section{General Terms}

Artificial Neural Networks, Pattern Mapping

\section{Keywords}

Neural networks, Multilayered feed-forward neural network (MLFNN), Bidirectional Associative Memory (BAM), function approximation

\section{INTRODUCTION}

Function approximation is the task of learning or constructing a function that generates approximately the same outputs from input vectors as the process being modeled, based on available training data. The approximate mapping system should give an output which is fairly close to the values of the real function for inputs close to the current input used during learning. There exist multiple methods that have been established as function approximation tools, where an artificial neural network (ANNs) is the one which has been very popular recently. A trained neural network is expected to capture the system characteristics in their weights. Such network is supposed to have generalized from the training data, if for a new input the network produces the same output which the system would have produced. Function approximation is an instance of supervised learning, the primary topic studied in machine learning, artificial neural networks, pattern recognition, and statistical curve fitting. In principle, any of the methods studied in these fields can be used in reinforcement learning.

Multilayered feed-forward neural networks (MLFNNs) trained with back-propagation algorithm and the bidirectional associative memory (BAM) trained with Hebb rule are the two categories of ANN which have been extensively used for pattern mapping (and thus for function approximation) task [1-3]. Due to their design, MLFNN with hidden layers are generally used for the classification purposes and suffers with the problem of generalization when used for pattern mapping. To overcome the well-known ill-posed problem [Tikhonov and Arsenin, 1977], a radial basis function network (RBFN) and counter propagation neural network (CPN) has been used. The underlying algorithms which modify weights separate such networks from back propagation. In back-propagation, the error and corresponding weight modification are propagated backwards from the output layer to the input layer. Different training samples may exert opposite forces in increasing or decreasing the same weight, and the net effect is that weight changes are often of extremely small magnitude in networks with learning rates small enough to have some assurance of converging to a local minimum of the mean squared error.

\section{RELATED WORK}

Substantial work has been cited in the literature for function approximation using feed-forward neural networks (FNNs) (Cybenko, 1989; Hecht-Nielsen, 1989; Carroll and Dickinson, 1989; Hornik, 1990, 1993; Park and Sandberg, 1991, 1993; Barron, 1993). FNNs are established structures capable of approximating generic classes of functions, including continuous and bounded. The structure studied varied in the terms of number of hidden layers for a particular function and different classes of FNNs were defined for approximating different classes of functions as per a set of approximation criteria. It is well known that a two-layered FNN, i.e. one that does not have any hidden layers, is not capable of approximating generic nonlinear continuous functions (Widrow, 1990). On the other hand, five or more layer FNNs are rarely used in practice. Hence, almost all the work deals with the most challenging issue of the approximation capability of three/four-layered FNNs. A three layered architecture was explored in [4] and the results obtained remained interesting.

Multilayer feed-forward neural networks have simpler dynamics compared to feedback or recurrent neural networks [5-7] and BAMs. BAMs has limited storage and mapping capability due to the use of Hebbian learning rule. There has been reported substantial work [8]-[25] in the literature to overcome this limitation. In the high-order BAM [8], highorder nonlinearity is applied to forward and backward information flows to increase the memory capacity and improve error correction capability. The exponential BAM [9] employs an exponential scheme of information flow to exponentially enhance the similarity between an input pattern 
and it's nearest stored pattern. It improves the storage capacity and error correcting capability of the BAM, and has a good convergence property. In [10], a high capacity fuzzy associative memory (FAM) for multiple rule storage is described. A weighted-pattern learning algorithm for BAM is described in [11] by means of global minimization. As inspired by the perceptron-learning algorithm, an optimal learning scheme for a class of BAM is advanced [12]-[13], which has superior convergence and stability properties. In [14], the synthesis problem of bidirectional associative memories is formulated as a set of linear inequalities that can be solved using the perceptron training algorithm. A multilayer recursive neural network with symmetrical interconnections is introduced in [15] for improved recognition performance under noisy conditions and for increased storage capacity. In [16], a new bidirectional heteroassociative memory is defined which encompasses correlational, competitive and topological properties and capable of increasing its clustering capability. Other related work can be found in [17]-[20] in which either by adding dummy neuron, increasing in number of layers or manipulating the interconnection among neurons in each layer, the issue of performance improvement of BAM is addressed. Even some new learning algorithms were introduced to improve the performance of original BAM [21][25].

\section{PROPOSED MODEL}

Various algorithms used in the methods discussed in section 2 , work only in one direction i.e. forward direction and the error thus calculated is propagated backward and weights are adjusted accordingly. A new method based on Back Propagation (BP) algorithm is proposed. This works in two phases: forward phase and backward phase (now onwards Phase-I and Phase-II). Since in both the phases the error is calculated and weights are adjusted accordingly, once in forward direction and then in backward direction, therefore there is a significant improvement in convergence. In this section outline of the theory behind the proposed approach is presented.

\subsection{Bidirectional Associative Memory}

In Bidirectional associative memory, neurons in one layer are fully connected to the neurons of second layer. It is a heteroassociative, content-addressable memory.

If a feed-forward network with two hidden layers is created and connections among various neurons of these layers are done in both directions as shown in Figure $\mathbf{1}$ and error minimization using back propagation algorithm is performed on this architecture, then this architecture corresponds to the BAM. Here the weights are taken symmetrical, means

$$
W_{i j}=W_{j i} \quad \text { for any two nodes } i \text { and } j
$$

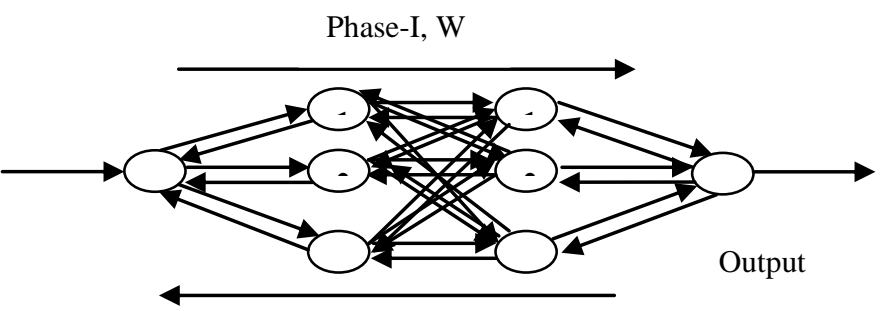

Fig 1: A typical 1- $n_{1}-n_{2}-1$ feed-forward architecture as Bidirectional Associative Memory

\subsection{The standard BP Algorithm for $1-n_{1}-n_{2}-$ 1 feed-forward architecture}

Phase-II, W'

- $\quad$ Create a feed-forward network with one input, ' $h$ ' hidden units and one output.

- $\quad$ Initialize all the weights to small random values.

- $\quad$ Repeat until $\left(\mathrm{E}_{\mathrm{avg}}=\sum_{k=1}^{Q} \mathrm{E}_{\mathrm{k}}<\tau\right)$ where $\boldsymbol{\tau}$ is the tolerance level of error

1. Input pattern $X_{k}$ from training set and compute the corresponding output $S_{y}$

2. For corresponding output, calculate the error as $\delta_{o}=S_{y}\left(D-S_{y}\right)\left(1-S_{y}\right)$

3. For each hidden unit ' $h$ ', calculate

$$
\delta_{h}=S_{h}\left(1-S_{y}\right) W_{h j} \delta_{o}
$$

4. Update each network weight between hidden and output layer $W_{h j}$ as follows:

$$
\begin{aligned}
& \qquad W_{h j}=W_{h j}+\eta \Delta W_{h j}+\alpha W_{h j} \text { old } \\
& \text { where, } \quad W_{h j}=S_{y} * \delta_{o} \\
& \text { and } \quad W_{h j} \text { old }=\Delta W_{h j}
\end{aligned}
$$

5. Update each network weight between input and hidden layer $W_{\text {ih }}$ as follows:

$$
\begin{aligned}
W_{i h} & =W_{i h}+\eta \Delta W_{i h}+\alpha W_{i h} \text { old } \\
\text { where } \quad W_{i h} & =\delta_{h} * X_{k} \\
\text { and } \Delta W_{i h} \text { old } & =\Delta W_{i h}
\end{aligned}
$$

\subsection{The proposed two- phase BP Algorithm}

Phase-I (forward phase):

The standard BP algorithm

Phase-II (backward phase):

- $\quad$ Repeat until $\left(\mathrm{E}_{\mathrm{avg}}=\sum_{k=1}^{Q} \mathrm{E}_{\mathrm{k}}<\tau\right)$ where $\tau$ is the tolerance level of error

1. $\quad W_{h i}=W_{i h}^{\prime}$ and $W_{j h}=W_{j h}^{\prime}$

2. Input pattern $D_{k}$ for corresponding $X_{k}$ and compute the corresponding output $S_{u}$

3. For corresponding output $S_{u}$, calculate the error as

$$
\delta_{i}=S_{u}\left(X-S_{u}\right)\left(1-S_{u}\right)
$$

4. For each hidden unit ' $h$ ', calculate

$$
\delta_{h}=S_{h}\left(1-S_{h}\right) W_{h i} \delta_{i}
$$

5. Update each network weight between hidden and input layer $W_{\text {hi }}$ as follows:

$$
W_{h i}=W_{h i}+\eta \Delta W_{h i}+\alpha W_{h i} \text { old }
$$


where, $W_{h i}=S_{h} * \delta_{i}$ and $W_{h i}$ old $=\Delta W_{h i}$

6. Update each network weight between output and hidden layer $W_{j h}$ as follows:

$$
W_{j h}=\Delta W_{j h}+\eta \Delta W_{j h}+\alpha W_{j h} \text { old }
$$

where, $\Delta W_{j h}=\delta_{i} * D_{k}$ and $\Delta W_{j h}$ old $=\Delta W_{j h}$

\section{SIMULATION DESIGN EXPERIMENTS}

AND

A four-layered feed-forward neural network has been created for the experimentation. At the input and output layers, only one neuron has been considered since the function $\{\sin (x)\}$ under consideration is a univariate function. Since there does not exist any thumb rule as far as the number of hidden layers and number of neurons therein. It is also well known that a two-layered FNN, i.e. one that does not have any hidden layers, is not capable of approximating generic nonlinear continuous functions (Widrow, 1990). On the other hand, five or more layer FNNs are rarely used in practice. Hence, almost all the work deals with the most challenging issue of the approximation capability of three/four-layered FNN. Various combinations of neurons ranging from 2 to 35 in both hidden layers are experimented. The summary of the various parameters like the learning rate, momentum etc. used is shown in Table 1.

At different learning rates and fixed momentum value (0.6), the convergence of the function has been studied with tolerance value .005 by both algorithms discussed in section $3.2 \& 3.3$ - standard back propagation and the proposed twophase back propagation algorithm (which let the MLFNN work as BAM). For each architecture five runs for both the above algorithms were executed and the average number of epochs was recorded at which the convergence was achieved for the taken error tolerance. The results have been tabulated in Table 2 (a), (b) and (c). One epoch in standard BP algorithm means when all the training patterns are presented once, while one epoch in proposed two-phase BP algorithm means when for all patterns both forward and backward phases run once. The same results has been shown graphically in Figure 2.

Table 1: The parameters used in simulation.

\begin{tabular}{|c|c|}
\hline Parameters & Number/Values \\
\hline Input Neurons & 1 \\
\hline Hidden Neurons in hidden layer 1 $\left(\boldsymbol{n}_{1}\right)$ & $2 \ldots \ldots 35$ \\
\hline Hidden Neurons in hidden layer 2 $\left(\boldsymbol{n}_{2}\right)$ & $2 \ldots \ldots 35$ \\
\hline Number of Patterns & 5 \\
\hline Learning rate, $\boldsymbol{\eta}$ & $0.7 / 0.8 / 0.9 / 1.0$ \\
\hline Momentum, $\boldsymbol{\alpha}$ & 0.6 \\
\hline Tolerance, $\boldsymbol{\tau}$ & 0.005 \\
\hline
\end{tabular}

Table 2: Number of epochs required for convergence for approximating the function $y=\sin (x)$

(a) Case I: $1-n_{1}-n_{2}-1$, where $n_{1}<n_{2}$

\begin{tabular}{|c|c|c|c|c|c|c|c|c|}
\hline \multirow{3}{*}{ 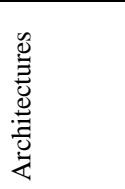 } & \multicolumn{8}{|c|}{ Learning rates } \\
\hline & \multicolumn{2}{|c|}{$\eta=0.7$} & \multicolumn{2}{|c|}{$\eta=0.8$} & \multicolumn{2}{|c|}{$\eta=0.9$} & \multicolumn{2}{|c|}{$\eta=1.0$} \\
\hline & FFN & BAM & FFN & BAM & FFN & BAM & FFN & BAM \\
\hline 1-2-3-1 & 9692 & 1191 & 5467 & 857 & 6459 & 1048 & 5600 & 901 \\
\hline 1-2-4-1 & 7016 & 919 & 4847 & 910 & 3292 & 1121 & 8426 & 911 \\
\hline $1-2-5-1$ & 4837 & 965 & 4842 & 798 & 3573 & 714 & 4046 & 863 \\
\hline $1-3-4-1$ & 5238 & 738 & 3908 & 667 & 4260 & 597 & 5120 & 692 \\
\hline $1-3-5-1$ & 4284 & 734 & 4758 & 657 & 8299 & 569 & 5717 & 631 \\
\hline 1-4-5-1 & 3443 & 687 & 3813 & 583 & 4257 & 489 & 2817 & 537 \\
\hline $1-5-10-1$ & 2242 & 508 & 3331 & 523 & 2543 & 411 & 1997 & 417 \\
\hline 1-10-15-1 & 1267 & 375 & 1013 & 372 & 1713 & 340 & 1042 & 270 \\
\hline $1-15-20-1$ & 843 & 313 & 816 & 255 & 690 & 246 & 632 & 251 \\
\hline $1-20-25-1$ & 678 & 235 & 560 & 236 & 591 & 203 & 473 & 223 \\
\hline $1-25-30-1$ & 542 & 227 & 606 & 203 & 520 & 200 & 463 & 191 \\
\hline $1-30-35-1$ & 576 & 204 & 540 & 295 & 448 & 185 & 511 & 364 \\
\hline
\end{tabular}

(b) Case II: $1-n_{1}-n_{2}-1$, where $n_{1}>n_{2}$

\begin{tabular}{|c|c|c|c|c|c|c|c|c|}
\hline \multirow{3}{*}{ 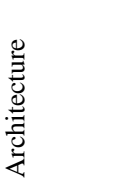 } & \multicolumn{8}{|c|}{ Learning rates } \\
\hline & \multicolumn{2}{|c|}{$\eta=0.7$} & \multicolumn{2}{|c|}{$\eta=0.8$} & \multicolumn{2}{|c|}{$\eta=0.9$} & \multicolumn{2}{|c|}{$\eta=1.0$} \\
\hline & FFN & BAM & FFN & BAM & FFN & BAM & FFN & $\begin{array}{c}\mathrm{BA} \\
\mathrm{M}\end{array}$ \\
\hline 1-3-2-1 & 5472 & 1146 & 5109 & 1490 & 8954 & 765 & 3315 & 125 \\
\hline 1-4-2-1 & 5201 & 1466 & 8167 & 779 & 3214 & 954 & 3913 & 925 \\
\hline 1-4-3-1 & 4054 & 774 & 4576 & 1034 & 6044 & 745 & 3408 & 549 \\
\hline $1-5-2-1$ & 4156 & 767 & 4369 & 898 & 6536 & 743 & 2457 & 760 \\
\hline $1-5-3-1$ & 3144 & 760 & 5785 & 729 & 2850 & 718 & 3399 & 728 \\
\hline 1-5-4-1 & 5673 & 732 & 4789 & 722 & 4432 & 626 & 2337 & 715 \\
\hline 1-10-5-1 & 1625 & 464 & 1578 & 488 & 1475 & 426 & 1489 & 442 \\
\hline 1-15-10-1 & 1204 & 355 & 886 & 362 & 1090 & 294 & 808 & 276 \\
\hline $1-20-15-1$ & 799 & 274 & 741 & 298 & 583 & 269 & 687 & 228 \\
\hline 1-25-20-1 & 688 & 270 & 521 & 242 & 548 & 201 & 436 & 213 \\
\hline $1-30-25-1$ & 571 & 229 & 461 & 223 & 466 & 200 & 374 & 192 \\
\hline 1-35-30-1 & 493 & 287 & 431 & 275 & 407 & 232 & 419 & 363 \\
\hline
\end{tabular}


(c) Case III: $1-n_{1}-n_{2}-1$, where $n_{1}=n_{2}$

\begin{tabular}{|c|c|c|c|c|c|c|c|c|}
\hline \multirow{3}{*}{ 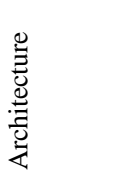 } & \multicolumn{8}{|c|}{ Learning rates } \\
\hline & \multicolumn{2}{|c|}{$\eta=0.7$} & \multicolumn{2}{|c|}{$\eta=0.8$} & \multicolumn{2}{|c|}{$\eta=0.9$} & \multicolumn{2}{|c|}{$\eta=1.0$} \\
\hline & FFN & BAM & FFN & BAM & FFN & BAM & FFN & BAM \\
\hline $1-2-2-1$ & 7993 & 1186 & 7124 & 1311 & 8473 & 906 & 6202 & 1123 \\
\hline $1-3-3-1$ & 4134 & 982 & 5613 & 804 & 3778 & 807 & 4609 & 658 \\
\hline $1-4-4-1$ & 5614 & 806 & 5550 & 635 & 5008 & 669 & 3556 & 567 \\
\hline $1-5-5-1$ & 4168 & 560 & 2269 & 581 & 3138 & 561 & 2122 & 499 \\
\hline $1-10-10-1$ & 1986 & 420 & 1202 & 403 & 1425 & 363 & 1058 & 345 \\
\hline $1-15-15-1$ & 986 & 353 & 903 & 308 & 685 & 268 & 679 & 255 \\
\hline $1-20-20-1$ & 757 & 267 & 703 & 246 & 614 & 227 & 533 & 191 \\
\hline $1-25-25-1$ & 819 & 211 & 537 & 223 & 485 & 213 & 456 & 185 \\
\hline $1-30-30-1$ & 522 & 219 & 516 & 204 & 488 & 198 & 445 & 182 \\
\hline $1-35-35-1$ & 446 & 244 & 437 & 287 & 482 & 346 & 513 & 307 \\
\hline
\end{tabular}

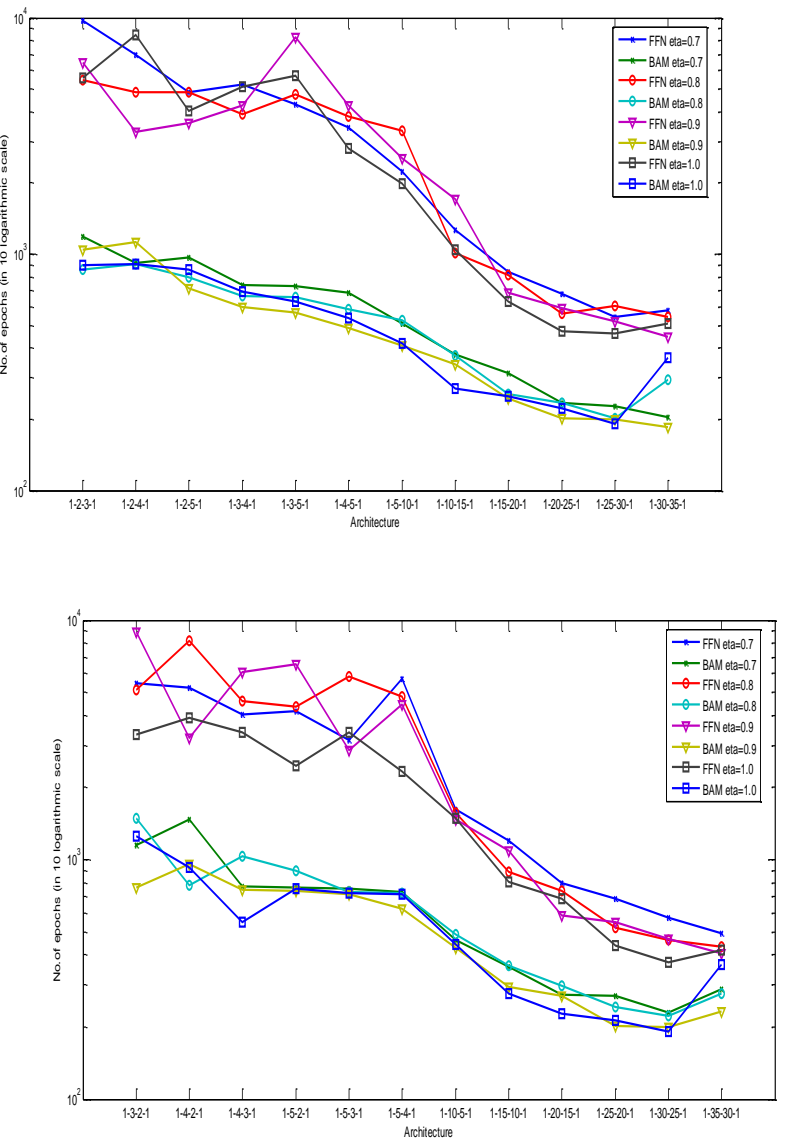

\section{RESULTS AND DISCUSSION}

The results shown in Table 2(a), (b), and (c) clearly indicate that the proposed two-phase back propagation algorithm for BAM architecture, in general, outperform the MLFNN architecture with standard back propagation algorithm. Table 2(a) shows the results for the architectures where first hidden layer has lesser number of neurons compared to the second hidden layer (1-n $\mathbf{n}_{\mathbf{1}}-\mathbf{n}_{\mathbf{2}} \mathbf{- 1}$, where $\left.\mathbf{n}_{\mathbf{1}}<\mathbf{n}_{\mathbf{2}}\right)$, while Table 2(b) contains for the other case where the number of neurons in first hidden layer are greater than those of second hidden layer $\left(\mathbf{1}-\mathbf{n}_{1}-\mathbf{n}_{2}-\mathbf{1}\right.$, where $\left.\mathbf{n}_{1}>\mathbf{n}_{2}\right)$ while Table 2(c) is for the case (1$\mathbf{n}_{1}-\mathbf{n}_{2}-\mathbf{1}$, where $\left.\mathbf{n}_{1}=\mathbf{n}_{2}\right)$. In every case, the number of epochs taken for the convergence of the taken function is substantially low in the case of proposed two-phase BP algorithm, in almost every architecture studied. It is observed that the number of epochs kept on lessening when one move on increasing the number of neurons in the hidden layers and various combinations are explored. And the best architecture is when the number of neurons in the two hidden layers is between 30 and 35, which can be seen that at the architecture 1-35-35-1, the number of epochs required for convergence starts increasing. Also there are some architectures in between where a larger number of epochs were recorded for convergence (e.g. 1-2-4-1, 1-4-2-1 etc.). Since both the algorithms work on the randomness of initial weight matrices, this may be one of the reasons for such results. Another obvious observation comes out that on increasing the learning rate from 0.7 to 1.0 the convergence is being achieved in lesser number of epochs, in general.

The results show that the best suited BAM architecture for the taken function $\sin (x)$ is $1-30-30-1$ with learning rate 1.0 and momentum 0.6.

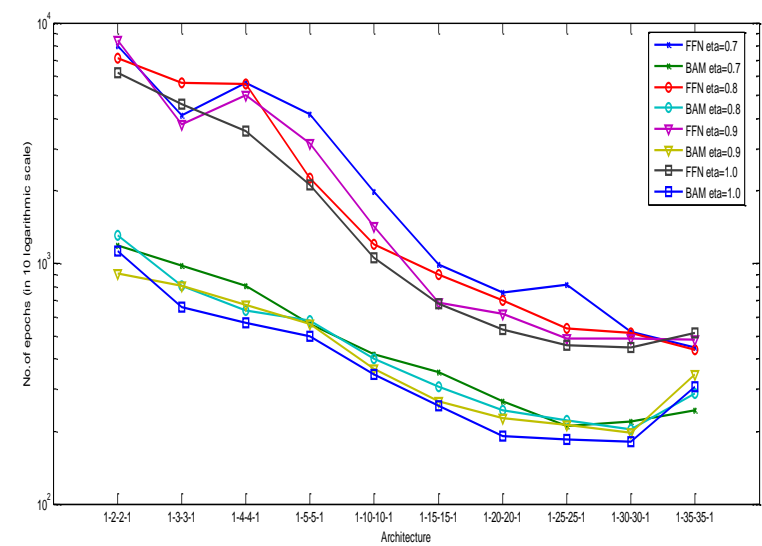

Fig. 2: Comparative graphs of the number of epochs required for convergence in MLFNNS and BAM for the architectures (i) $1-\mathbf{n}_{1}-\mathbf{n}_{2}-1$, where $\mathbf{n}_{1}<\mathbf{n}_{2}$ (ii) $1-\mathbf{n}_{1}-\mathbf{n}_{2}-1$, where $n_{1}>n_{2}$ (iii) $1-n_{1}-n_{2}-1$, where $n_{1}=n_{2}$ 


\section{CONCLUSION AND FUTURE SCOPE}

A new variation of back propagation algorithm, the TwoPhase BP, has been presented which when applied to multilayered feed-forward neural network (MLFNN) let it behave like bidirectional associative memory (BAM). The fourlayered architectures having various combinations of neurons in the two hidden layers have been experimented for a single valued-function. The results presented above suggest that proposed algorithm is better suited for function approximation and results obtained are quite encouraging. In future, the proposed algorithm can be applied for approximating variety of functions and the detailed results thus obtained may be compared with traditional algorithms and the effect on convergence could be studied.

\section{REFERENCES}

[1] Rumelhan D.E., Hinton G.E., and Williams R. J., "Learning Internal Representations by Error Propagation", Parallel Distributed Processing, Vol. I Foundations, MIT Press, Cambridge, MA, pages 318364. 1986.Ding, W. and Marchionini, G. 1997 A Study on Video Browsing Strategies. Technical Report. University of Maryland at College Park.

[2] Tang C. Z. and Kwan H. K., "Parameter effects on convergence speed and generalization capability of backpropagation algorithm". International Journal of Electronics, 74(1): 35-46, January 1993.

[3] Kosko B., Neural Networks and Fuzzy Systems, Prentice-Hall. Inc., New Jersey, 1992.

[4] Singh, M. and Kumar, S., "Using Multi-layered Feedforward Neural Network (MLFNN) Architecture as Bidirectional Associative Memory (BAM) for Function Approximation", IOSR Journal of Computer Engineering (IOSR_JCE), vol. 13, Issue 4, pp 34-38

[5] Pearlmutter B.A., "Gradient calculations for dynamic recurrent neural networks: a survey". IEEE Trans. On Neural Networks, 6 (5):1212-1228, Sept. 1995.Forman, G. 2003. An extensive empirical study of feature selection metrics for text classification. J. Mach. Learn. Res. 3 (Mar. 2003), 1289-1305.

[6] Prokhorov D.V., Feldkamp L.A., and Tyukin I.Y., "Adaptive behavior with fixed weights in RNN: an overview". Proceedings of International Joint Conference on Neural Networks, 2002, pages 2018 2022.

[7] Kwan H. K. and Yan J., "Second-order recurrent neural network for word sequence learning", Proceedings of 2001 International Symposium on Intelligent Multimedia, Video and Speech Processing, Hong Kong, May 2-4. 2001, pages 405-408.

[8] Tai H.-M.. Wu C.-H., and Jong, T.-L., 'High-order bidirectional associative memory': Elecrronics krlers, 25(21):1424-1425, 12th Oct. 1989.

[9] Jeng Y.-J., Yeh C.-C., and Chiueh T.D., 'Exponential bidirectional associative memories", Electronics Letters, 26(11):717-718,24 ${ }^{\text {th }}$ May1990.
[10] Chung F.L. and Lee Tong, 'On fuzzy associative memory with multiple-rule storage capacity". IEEE Trans. on Fuzzy Systems, 4(3):375-384, August 1996.

[11] Wang T.. Zhuang X., and Xing X., 'Weight learning of bidirectional associative memories by global minimization", IEEE Trans. on Neural Networks, 3(6):1010-1018, Nov. 1992.

[12] Shanmukh K. and Venkatesh Y.V., 'Generalized scheme for optimal learning in recurrent neural networks': IEE Proc: Vision, Image, and Signal Processing. 142(2):71-77, April 1995.

[13] Salih, I. Smith, S.H., and Liu, D., 'Design of bidirectional associative memories based on the perceptron training technique". Proceedings of IEEE International Symposium on Circuits and Systems, 1999, Vol. 5, pages 355-358.

[14] Kwan H. K. and Tsang P. C., 'Multi -layer recursive neural network': Proceedings of Canadian Conference on Electrical and Computer Engineering, Montreal, Canada, September 17-20, 1989, Vol. 1. pages 428-431.

[15] Widrow B. and Winter R., 'Neural Nets for Adaptive Filtering and Adaptive Pattern Recognition", IEEE Computer Magazine, pages 25-39. March 1988.

[16] Chartier S., Giguere G. and Langlois D., "A new bidirectional heteroassociative memory encompassing correlational, competitive and topological properties", Neural Networks 22 (2009), pp 568-578, 2009

[17] Wang Y.F., Cruz J.B. and Mulligan J.H., "Two coding strategies for bidirectional associative memory", IEEE Trans. Neural Networks, vol. 1, no. 1, pp 81-92, 1990

[18] Wang Y.F., Cruz J.B. and Mulligan J.H., "Guaranteed recall of all training pairs for bidirectional associative memory", IEEE Trans. Neural Networks, vol. 2, no. 6 , pp 559-567, 1991

[19] Kang H., "Multilayer associative neural network (MANN's): Storage capacity versus perfect recall", IEEE Trans. Neural Networks, vol. 5, pp 812-822, 1994

[20] Wang Z., "A bidirectional associative memory based on optimal linear associative memory", IEEE Trans. Neural Networks, vol.45, pp 1171-1179, Oct.1996

[21] Hassoun M.H. and Youssef A.M., "A high performance recording algorithm for Hopfield model associative memories", Opt. Eng., vol. 27, no. , pp 46-54, 1989

[22] Simpson P.K., "Higher-ordered and interconnected bidirectional associative memories", IEEE Trans. Syst. Man. Cybern., vol. 20, no. 3, pp 637-652, 1990

[23] Zhuang X., Huang Y. and Chen S.S., "Better learning for bidirectional associative memory", Neural Networks, vol.6, no.8, pp1131-1146, 1993

[24] Oh H. and Kothari S.C., "Adaptation of the relaxation method for learning bidirectional associative memory", IEEE Trans. Neural Networks, vol.5, pp 573-583, July 1994.

[25] Wang C.C. and Don H.S., "An analysis of high-capacity discrete exponential BAM", IEEE Trans. Neural Networks, vol. 6, no. 2, pp 492-496, 1995. 